\title{
Orte und Worte der Universität Ein Atlas
}

\author{
Erschienen in: WORTATLAS
}

Von: Julika Griem, Markus Steinmayr

Die Art und Weise, wie über Universität, Studium und Lehre, Wissenschaft und Forschung gesprochen wird, ist immer auch ein Ausdruck unserer Vorstellungen über diese Gegenstände. Worte wie „Wettbewerb“, „Exzellenz“, „Digitalisierung“, „Innovation“, „Diversität“ und viele andere prägen unsere alltägliche Kommunikation und die Spielräume, in denen das Wissenschaftssystem sich beschreiben, darstellen und verstehen lassen kann. Unsere Wahrnehmung ist in vielen Situationen so stark von öffentlich und ökonomisch einsetzbaren Schlagworten bestimmt, dass wir aus den Augen verlieren, ob noch besser pointiert und differenziert werden könnte. Gleichzeitig können wir nicht auf wirkmächtige Schlüsselbegriffe verzichten, um effektiv zu kommunizieren.

Wir möchten, gemeinsam mit Ihnen, jene Worte sammeln, die den Wissenschaftsalltag prägen, und darüber ins Gespräch und zu treffenderen Beschreibungen und Analysen kommen. Mit der Etymologie, der Semantik und der Pragmatik dieser Worte interessiert uns, was sie tun, wenn wir sie benutzen - mit der Universität und uns allen. Welche Vorstellungen entstehen und werden vorausgesetzt? Woran schließen spezifische Worte an, was schließen sie aus? Wen sprechen sie an, wen erreichen sie nicht? Und in welchen textuellen, medialen und institutionellen Umgebungen gewinnen sie ihre Aussagekraft - oder verlieren diese auch?

Im besten Fall könnte sich unsere Suche nach Schlüsselworten wissenschaftlicher und wissenschaftspolitischer Selbstbeschreibung zu einer Sammlung auswachsen - ganz im Sinne Walter Benjamins, der in seinem Denkbild "Ich packe meine Bibliothek" schrieb, dass die Existenz des Sammlers „dialektisch gespannt zwischen den Polen der Unordnung und der Ordnung“ ist. Wir wollen mit Ihnen diese Spannung erzeugen und Unordnung in die Diskurse bringen, um die Worte, mit denen sie arbeiten, in neue, Erkenntnisse auslösende Sinnzusammenhänge zu bringen.

Die gemeinsam angelegte Sammlung soll unter dem Titel „WortAtlas der Universität“ auf diesem Blog erscheinen. Beginnen wollen wir mit einem kurzen Vorlauf, der sich weniger den Worten als den Orten der Universität widmet. Denn letztere lebt ja nicht nur von Diskursen und Symbolen, sondern auch von Materialitäten und physischen Räumen, in denen das stattfindet, was Universität ist oder sein könnte. Auch die Orte der Universität sind wie ihre Worte, historisch und vergleichend betrachtet, vielschichtig 
und vieldeutig. Sie sind, wie zum Beispiel die Bibliothek, Quellen von Sentimentalität, oder wie der Campus Stätten, die gerade zu verschwinden drohen. Sie sind aufgeladen mit affektiver Energie und somit gleichsam Medien der Selbstverständigung über das, was die Universität einmal war, ist oder sein könnte. Gerade die derzeitige Situation lässt uns die Räume und die Orte der Universität vermissen: die Seminarräume und die Bibliothek, aber auch den Flur, die Bänke im Innenhof und die Kaffeeküche; und vielleicht vermissen auch manche ihr Büro.

Bevor wir den "WortAtlas“ aufschlagen, soll es also, quasi in situierender Rückversicherung, um Orte und Räume gehen, an denen und mit denen man aus ikonographischer, raumtheoretischer, medientheoretischer symboltheoretischer, ethnologischer und weiterer Sicht die Universität (im Moment ihrer Virtualisierung) noch einmal etwas anders erfassen kann. Ob aus der gemeinsam auf diesem Blog angelegten Reihe noch anderes und mehr werden kann, liegt in der Hand derer, die sich hoffentlich von dieser Einladung angesprochen fühlen. Auch englischsprachige Beiträge sind willkommen.

SUGGESTED CITATION: Griem, Julika; Steinmayr, Markus: Orte und Worte der Universität. Ein Atlas, in: KWI-BLOG, [https://blog.kulturwissenschaften.de/orte-undworte-der-universitat/], 12.04.2021

DOI: https://doi.org/10.37189/kwi-blog/20210412-0800 


\section{DuEPublico}

Duisburg-Essen Publications online

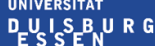

offen im Denken

Dieser Text wird via DuEPublico, dem Dokumenten- und Publikationsserver der Universität Duisburg-Essen, zur Verfügung gestellt. Die hier veröffentlichte Version der E-Publikation kann von einer eventuell ebenfalls veröffentlichten Verlagsversion abweichen.

DOI: $\quad$ 10.37189/kwi-blog/20210412-0800

URN: urn:nbn:de:hbz:464-20210412-105116-1

Alle Rechte vorbehalten. 